\title{
A CASE OF LEPROSY \\ IN A SEVEN MONTHS OLD CHILD
}

John A. Dreisbach, M.D.

The incubation period of leprosy is usually stated as being from 3-5 years up to IO-I5 years, or at times even longer. Workers with experience in highly endemic areas, however, occasionally see cases under three years of age with clinical leprosy. The youngest case I have noted in the literature is that of an eighteen months old child.

In January, I95I, a Hausa family of five presented themselves for examination and admission at the Kano Leprosy Settlement of the Sudan Interior Mission. Careful clinical and laboratory examinations were carried out. The mother showed no clinical or laboratory evidence of leprosy. The father was a very far advanced case of lepromatous leprosy, showing heavy nodulations and infiltrative lesions. There was marked mucous membrane involvement with labial, buccal, lingual, pharyngeal, and laryngeal nodules and infiltrations. There was nasal septal ulceration and perforation. There were three sons, of ages five years, three years, and seven months. All three had early incipient lesions manifested as flat, hypopigmented macules.

We shall confine this report to the youngest child. This baby was quite young and according to the parents was seven months old. The infant did not appear to be any older than this. The history was that, due to his condition, the father was unable to work the family farm and do the other tasks ordinarily performed by the man. As a result, the wife had to assume the responsibility of these tasks, and turned the care of the children almost completely over to the husband. He had had almost constant contact with this child from birth.

The parents state that they first noted the skin lesions, which are described later, two months previously, when the child was five months old. They stated that the lesions were larger and more noticeable at the time of admission.

\section{Physical Examination}

The patient was a well developed, well nourished male infant of seven months. The physical examination by systems was essentially negative, with the exception of the spleen, which was palpable two fingers' breadth below the left costal margin, and the skin lesions to be described.

There were three lesions noted upon admission. There was 
a large, flat, hypopigmented macular lesion of the mask area of the face. This was rather vague. A second lesion was noted on the posterior aspect of the left thigh, extending up on to the left buttock. This, likewise, was a flat, hypopigmented, macular lesion with vague margins. The third lesion was on the anterior aspect of the right thigh. It was a discreet, moderately hypopigmented, macular lesion with more definite margins and tending to slight central infiltrations.

The infant was too young to co-operate for a neurological examination. The lesion on the right thigh was subjected to the mecholyl test. There was a failure of response of the sweating mechanism in the lesion, whereas there was a normal active response in a control area of healthy skin.

The routine laboratory examinations were all within normal limits with the exception of the skin smears. A smear taken from the lesion on the right thigh revealed a few single Mycobacteria leprae. Smears taken from the other lesions and areas were all negative. The child was diagnosed as having macular leprosyincipient lesions of childhood. The child was admitted and treatment instituted.

The child was placed on sulphetrone, which was administered as a sterile $50 \%$ aqueous solution and given by subcutaneous injection. These injections were given twice weekly with a maximum weekly dose of one gram. The child has tolerated this treatment very well. There has been a mild anaemia throughout the period of treatment, but never marked enough to warrant discontinuing treatment.

At the end of the first year the child had an episode that probably was evidence of mild drug toxicity. It was manifested as objective pruritus. There were no other manifestitations. The child was given a rest of one month, after which treatment was started again and has continued uninterruptedly to the present.

By the end of the fifth month of treatment the lesions on the face and right thigh were completely repigmented. The lesion on the left thigh persisted for over a year. At this time, after 28 months of treatment, there are no clinical manifestations of activity of the disease. We plan to discharge the patient after 36 months of treatment if the disease is still clinically arrested.

With the exception of the admission skin smears, all of the smears have been negative for Mycobacteria leprae.

\section{SUMMARY}

A case is reported of a seven months old child with clinical leprosy. The clinical findings and response to sulphetrone treatment are discussed. 